\title{
Optochemical control of genetically engineered neuronal nicotinic acetylcholine receptors
}

\author{
Ivan Tochitsky ${ }^{1 \dagger}$, Matthew R. Banghart ${ }^{2 \dagger}$, Alexandre Mourot', Jennifer Z. Yao ${ }^{2}$, Benjamin Gaub ${ }^{3}$, \\ Richard H. Kramer ${ }^{1,3 \star}$ and Dirk Trauner ${ }^{2,4 \star}$
}

\begin{abstract}
Advances in synthetic chemistry, structural biology, molecular modelling and molecular cloning have enabled the systematic functional manipulation of transmembrane proteins. By combining genetically manipulated proteins with lightsensitive ligands, innately 'blind' neurobiological receptors can be converted into photoreceptors, which allows them to be photoregulated with high spatiotemporal precision. Here, we present the optochemical control of neuronal nicotinic acetylcholine receptors (nAChRs) with photoswitchable tethered agonists and antagonists. Using structure-based design, we produced heteromeric $\alpha 3 \beta 4$ and $\alpha 4 \beta 2$ nAChRs that can be activated or inhibited with deep-violet light, but respond normally to acetylcholine in the dark. The generation of these engineered receptors should facilitate investigation of the physiological and pathological functions of neuronal nAChRs and open a general pathway to photosensitizing pentameric ligand-gated ion channels.
\end{abstract}

A cetylcholine $(\mathrm{ACh})$ and its receptors have always been at the forefront of new developments in physiology. With its isolation as 'Vagusstoff' in 1921, Otto Loewi established that a small diffusible molecule could mediate nervous activity and shaped the concept of a neurotransmitter ${ }^{1}$. Nicotinic acetylcholine receptors (nAChRs) were among the first ion channels investigated, long before the advent of molecular cloning and heterologous expression $^{2}$. Numerous techniques in biophysics and chemical biology, including patch clamp electrophysiology ${ }^{3}$, cryoelectron microscopy ${ }^{4}$ and the expansion of the genetic code ${ }^{5}$, were developed using nAChRs, and the powerful methods of molecular cloning were applied to nAChRs early on ${ }^{6-8}$. These studies established that $\mathrm{nAChRs}$ are pentameric ligand-gated cation channels expressed throughout the mammalian nervous system and at the neuromuscular junction ${ }^{1}$. The neuronal nAChR subtypes are composed of $\alpha 2-\alpha 10$ and $\beta 2-\beta 4$ subunits and can assemble both as heteromeric (for example, $(\alpha 4)_{2}(\beta 2)_{3}$ ) and homomeric (for example, $(\alpha 7)_{5}$ ) pentamers ${ }^{1}$. Whereas the function of muscle nAChRs is well established, the physiological roles of neuronal nAChRs are still being unravelled. For instance, they are implicated strongly in the pathophysiology of several psychiatric disorders, as well as in nicotine addiction and Alzheimer's disease ${ }^{1,9}$. However, progress in this regard has been held back by the lack of subtype-selective nAChR pharmacology and the difficulties associated with selectively targeting $\mathrm{nAChRs}$ in different parts of the brain.

These issues can be addressed using an approach called 'optochemical genetics'10,11. In essence, this is an effort to photosensitize innately 'blind' receptors using synthetic photoswitches. These can be attached covalently as photoswitchable tethered ligands (PTLs), which require a reactive functional group for bioconjugation (typically the sulfhydryl group of a cysteine $\mathrm{e}^{12,13}$ ). By introducing the cysteine through genetic manipulation, these receptors can be targeted in a subtype-selective manner based on the subunit that contains the mutation ${ }^{14}$. Furthermore, the mutant receptors can be expressed in specific cell types in the brain and controlled with the millisecond precision and subcellular spatial resolution that only light can provide. This has been achieved already with voltage-gated ion channels ${ }^{12,14}$ and glutamate receptors ${ }^{13,15}$. We now demonstrate that our approach towards photosensitizing nature's molecular machines can be applied to pentameric ligandgated ion channels as well, and introduce the genetically engineered, light-controlled nAChR (LinAChR).

\section{Results}

PTL design. The development of LinAChR first required the design and synthesis of appropriate PTLs. Our PTLs typically consist of a maleimide as the cysteine reactive group, an azobenzene photoswitch and a ligand head group that resembles known receptor agonists or antagonists. Three compounds known to interact with $n A C h R s$ guided the choice of the ligand: the photoaffinity label AC-5 (1), the agonist homocholine phenyl ether (HoChPE) (2) and the antagonist MG-624 (3). The presence of an aromatic ring separated by seven atoms from the ACh moiety in AC-5 (Fig. 1a), which acts as a full agonist at muscle nAChRs ${ }^{16}$, suggests that the steric bulk of an azobenzene photoswitch may be tolerated in PTLs that act as tethered agonists. Although the much shorter molecule HoChPE is an agonist of $\mathrm{nAChRs}{ }^{17,18}$, the stilbene derivative MG-624 is a potent antagonist of neuronal receptors ${ }^{19}$. Therefore, on the basis of the steric similarity of stilbenes and azobenzenes, we anticipated that corresponding PTLs could function as antagonists. Based on these model compounds, we synthesized trans- and cis-maleimide-azobenzene-acylcholine (MAACh) (4a and $\mathbf{4 b}$, respectively) (Fig. 1a), a putative photoswitchable agonist for nAChR. We also synthesized trans- and cis-maleimide-azobenzenehomocholine (MAHoCh) (5a and $\mathbf{5 b}$, respectively) (Fig. 1b), wherein the choline moiety was replaced with homocholine and the spacer was removed to resemble MG-624. MAACh and MAHoCh were prepared by multistep total synthesis, as detailed in Supplementary Fig. S1. By taking absorbance measurements of the ligands in solution, we determined that both molecules can be

\footnotetext{
'Department of Molecular and Cell Biology, University of California, Berkeley, California 94720, USA, ${ }^{2}$ Department of Chemistry, University of California, Berkeley, California 94720, USA, ${ }^{3}$ Helen Wills Neuroscience Institute, University of California, Berkeley, California 94720, USA, ${ }^{4}$ Department of Chemistry, Ludwig-Maximilians-Universität, München and Center for Integrated Protein Science, 81377 Munich, Germany; ${ }^{\dagger}$ These authors contributed equally to this work. `e-mail: rhkramer@berkeley.edu; dirk.trauner@lmu.de
} 
a<smiles>CN(C(=O)NCCCCCC(=O)OCC[N+](C)(C)C)c1ccc([N+]#N)cc1</smiles><smiles>C[N+](C)(C)CCOC(=O)CCCCCNC(=O)Nc1ccc(N=Nc2ccc(NC(=O)CN3C(=O)C=CC3=O)cc2)cc1</smiles>

$4 a$ trans-MAACh

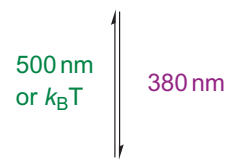<smiles>C[N+](C)(C)CCOC(=O)CCCCCNC(=O)Nc1ccc(/N=N\c2ccc(NC(=O)CN3C(=O)C=CC3=O)cc2)cc1</smiles>

c

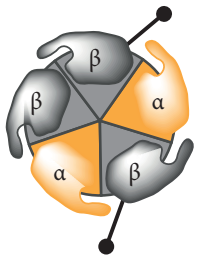

$380 \mathrm{~nm}$

$500 \mathrm{~nm}$ or $k_{\mathrm{B}} T$

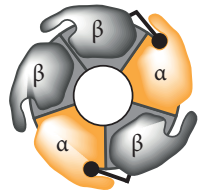

b<smiles>C[N+](C)(C)CCCOc1ccccc1</smiles><smiles>CC[N+](CC)(CC)CCOc1ccc(/C=C/c2ccccc2)cc1</smiles>

3

MG-624<smiles>C[N+](C)(C)CCCOc1ccc(N=Nc2ccc(NC(=O)CN3C(=O)C=CC3=O)cc2)cc1</smiles>

trans-MAHoCh
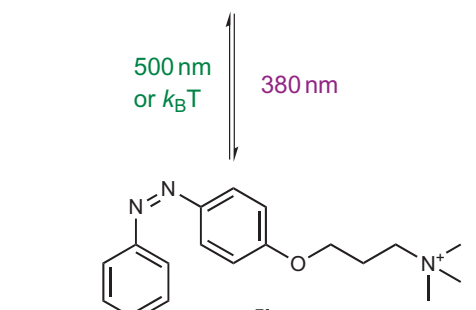

$5 b$<smiles>CNC(=O)CN1C(=O)C=CC1=O</smiles>

cis-MAHoCh d

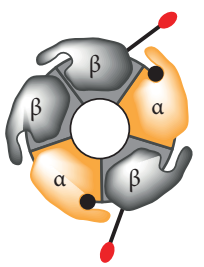

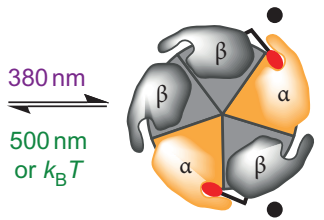

Figure 1 | Photoswitchable ligand design for the optical control of neuronal nAChRs. a,b, Structures of AC-5 and MAACh (a) as well as HoChPE, MG-624 and MAHoCh (b). The PTL molecules photoisomerize from trans to cis on illumination with $380 \mathrm{~nm}$ light and revert to trans under $500 \mathrm{~nm}$ light or in the dark. c, A tethered agonist is converted from its trans- into its cis-configuration under $380 \mathrm{~nm}$ light, and thus activates heteropentameric nAChRs. Illumination with $500 \mathrm{~nm}$ light or thermal relaxation deactivates the receptor. $\mathbf{d}$, A tethered antagonist is converted from its trans- into its cis-configuration under 380 nm light, which blocks heteromeric nAChRs from binding to ACh (black circles). Illumination with $500 \mathrm{~nm}$ light or thermal relaxation unblocks the receptor, which allows it to be activated by the neurotransmitter. $k_{\mathrm{B}}=$ Boltzmann constant.

converted maximally into their cis-isomer by illumination with $380 \mathrm{~nm}$ light and can then be reset to the trans-isomer by $500 \mathrm{~nm}$ illumination or thermal relaxation in the dark (Supplementary Fig. S2).

Preferably, a photosensitive receptor would be inactive in the dark, where azobenzene photoswitches generally reside in a transconfiguration (Fig. 1c). Illumination with $380 \mathrm{~nm}$ light would produce the cis-configuration of the azobenzene and activate the receptor. Deactivation could then be achieved through conversion of the photoswitch back to its inactive trans-configuration by illumination with $500 \mathrm{~nm}$ or by thermal relaxation in the dark. Similarly, a tethered antagonist should block the receptor in its cis-configuration (that is, at $380 \mathrm{~nm}$ ), but should leave the receptor unimpaired in its trans-configuration (at $500 \mathrm{~nm}$ or in the dark), allowing it to be activated by free ACh (Fig. 1d).

Attachment-site screening. These functional requirements can be met by the proper choice of cysteine attachment sites, which we decided to place on the $\beta 2$ or $\beta 4$ subunits of heteropentameric neuronal nAChRs. Using the X-ray structure of an acetylcholinebinding protein $(\mathrm{AChBP})$ in complex with carbamylcholine (PDB ID:1uv6 $)^{20}$ and the model of AC-5 docked into the Torpedo nAChR, we identified an antiparallel beta sheet in the $\beta$-subunit that faces the ligand-binding site as a potential region for PTL attachment ${ }^{16}$. Based on the calculated structures of our PTLs and distance measurements in the protein structures, we decided to 
a

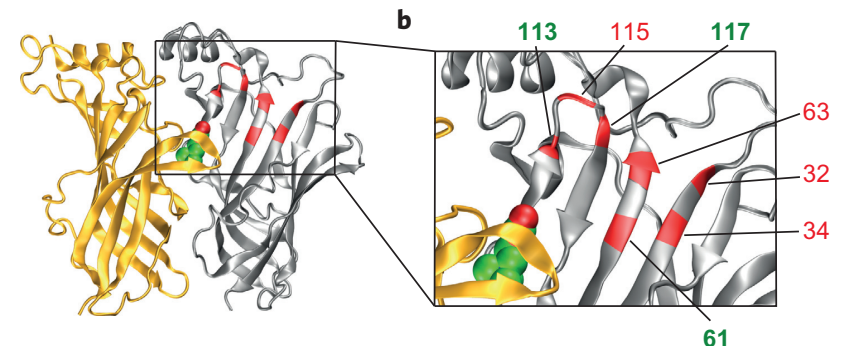

c

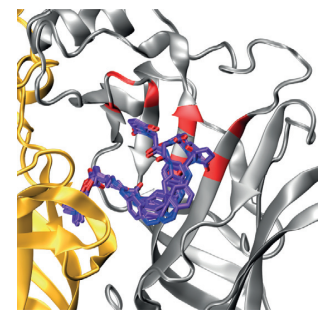

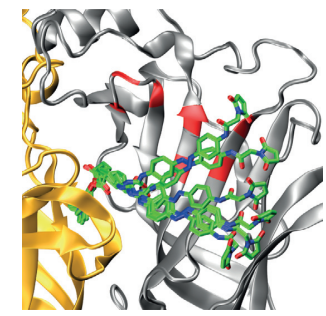

Figure 2 | Choice of cysteine attachment sites for photoswitchable ligand conjugation. a,b, Homology model of the $\alpha 4 \beta 2 \mathrm{nAChR}$ with ACh (green and red spheres, respectively) docked in the binding site and the engineered cysteine mutants shown in red and numbered in the enlarged view of the receptor (b). Only one $\alpha / \beta$ ( $\alpha$, yellow; $\beta$, grey) interface is shown for clarity. c,d, Docking results with unconstrained cis-MAACh (violet) (c) and transMAACh (green) (d) in the $\alpha 4 \beta 2$ homology model. Six of the most energetically favourable conformations are shown.

install cysteines in positions S32, R34, E61, T63, R113, N115 and S117 of the $\beta 4$ nAChR subunit. Homologous residues with identical numbering were chosen for $\beta 2$. These residues are shown mapped onto a homology model of the $\alpha 4 \beta 2$ receptor $^{21}$ (PDB ID:1ole) (Figs.2a,b), based on the structure of AChBP (ref. 22). A sequence alignment that relates these positions on various complementary subunits to AChBP is also provided (Supplementary Fig. S3).

Our choice of cysteines was validated further by molecular docking studies, which were carried out using the same homology model (Fig. 2c). Unconstrained cis-MAACh and trans-MAACh were docked into the $\alpha 4 \beta 2$ model and the rotatable bonds in the ligand allowed to move freely. We found that the maleimide moiety of MAACh comes very close to several of the engineered cysteine mutants shown in Fig. $2 \mathrm{~b}$ in the cis-form of the azobenzene photoswitch, in particular to cysteines in positions 61, 63 and 117 (Fig. 2c), but extends past most of them in its trans-form (Fig. 2d). Molecular modelling studies with MAHoCh were not undertaken because of the lack of ligand similarity in the antagonist-bound AChBP structures ${ }^{23}$ and the absence of a homology model of an antagonist-bound heteromeric neuronal nAChR.

To test our predictions experimentally, we first introduced the seven independent cysteine mutations into the $\beta 4$ subunit. Then, we screened $\alpha 4 \beta 4$ mutant $\mathrm{nAChRs}$ expressed heterologously in Xenopus oocytes, because this particular heteropentamer desensitizes very slowly compared to nAChRs composed of other neuronal subunits ${ }^{24}$. The oocyte system was chosen for its robust expression of heteromeric nAChRs and the lack of endogenous receptors. By contrast, non-neuronal mammalian cell lines often express nAChRs poorly and many neuronal mammalian cell lines possess endogenous nAChRs that would complicate the screening process.

Using two-electrode voltage-clamp recordings, we screened these $\alpha 4 \beta 4$ mutants for cis-agonism by treating oocytes with MAACh in the dark and then looking for the induction of a current on illumination with $380 \mathrm{~nm}$ light. We identified three cysteine mutations on the $\beta 4$ subunit where MAACh could evoke a photoactivatable
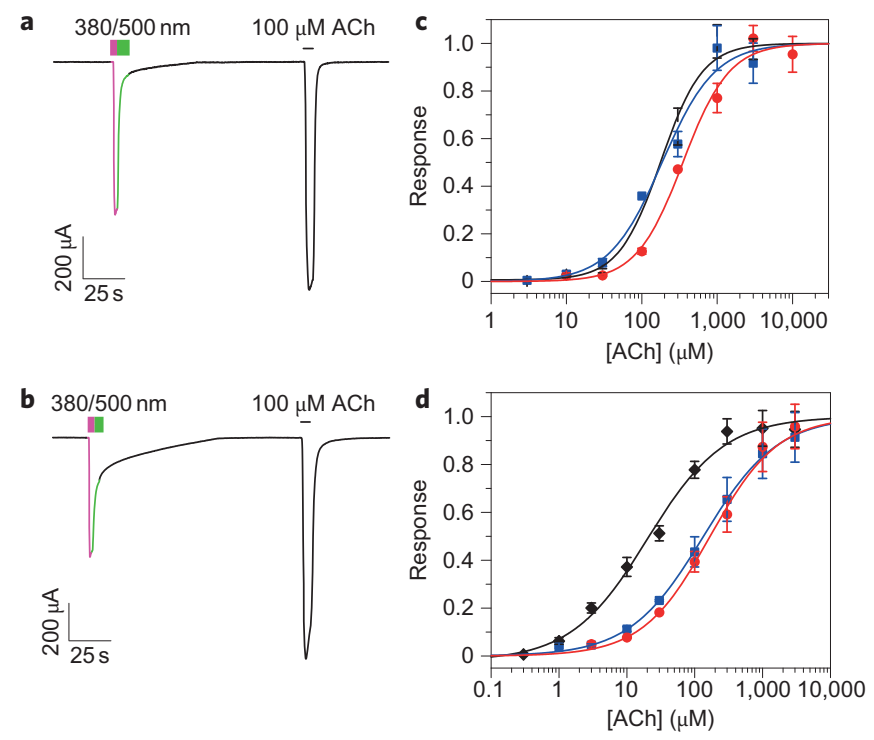

Figure 3 | Photoactivation of $n A C h R s$ with a tethered agonist. $\mathbf{a}, \mathbf{b}$, Photoactivation of the $\alpha 3 \beta 4 \mathrm{E} 61$ (a) and $\alpha 4 \beta 2 \mathrm{E} 61 \mathrm{C}$ (b) mutant receptors by tethered MAACh in Xenopus oocytes. Illumination at $380 \mathrm{~nm}$ (violet lines) triggers photoactivatable current and at $500 \mathrm{~nm}$ (green lines) shuts it off. c,d, ACh dose-response curves for the $\alpha 3 \beta 4$ (c) and the $\alpha 4 \beta 2$ (d) WT (black lines), E61C mutant (blue lines) and E61C mutant with tethered MAACh in the dark (red lines). Data are mean \pm s.e.m., $n=5$.

current in the cis- but not in the trans-configuration: E61C, $\mathrm{R} 113 \mathrm{C}$ and $\mathrm{S} 117 \mathrm{C}$. In the structure of carbamylcholine-bound AChBP (ref. 20) the distances from the alpha carbons of each of the three positions and the carbonyl carbon of carbamylcholine were very similar $(10.43 \AA, 11.28 \AA$ and $12.11 \AA$ for the E61C, $\mathrm{R} 113 \mathrm{C}$ and $\mathrm{S} 117 \mathrm{C}$ mutants, respectively). The remaining residues were farther away $(19.03 \AA, 14.28 \AA, 16.39 \AA$ and $16.54 \AA$ for S32C, R34C, T63C and N115C, respectively).

Photoactivation of engineered nAChRs. For a comprehensive electrophysiological analysis, these mutant $\beta 4$ subunits were expressed as $\alpha 3 \beta 4$ heteropentamers, which have a more established presence in the nervous system ${ }^{25}$. After labelling the $\alpha 3 \beta 4 E 61 C$ receptor with MAACh, illumination with $380 \mathrm{~nm}$ light produced an inward current that could be reversed with $500 \mathrm{~nm}$ light (Fig. 3a). The amplitude of the photoactivatable current was $6.8 \pm 1.3 \%$ (mean \pm standard error of the mean (s.e.m.), $n=10$ ) of the saturating cholinergic current and the receptor still responded normally to perfusion of $\mathrm{ACh}$ in the dark. Neither the E61C mutation nor MAACh conjugation greatly changed the halfmaximum effective concentration $\left(\mathrm{EC}_{50}\right)$ of the receptor for $\mathrm{ACh}$ (Fig. 3c, Supplementary Table S1). MAACh labelling at the other two residues, $\beta 4 \mathrm{R} 113 \mathrm{C}$ and $\beta 4 \mathrm{~S} 117 \mathrm{C}$, also produced photoactivatable currents of similar magnitude (Supplementary Fig. S4).

Next, we transposed the E61C mutant from the $\beta 4$ to the $\beta 2$ subunit and tested whether MAACh conjugation at the $\beta 2 \mathrm{E} 61 \mathrm{C}$ position could photoactivate the neuronal $\alpha 4 \beta 2$ receptor. Indeed, the MAACh-labelled $\alpha 4 \beta 2 \mathrm{E} 61 \mathrm{C}$ receptor also produced an inward current using $380 \mathrm{~nm}$ light, which could be terminated with $500 \mathrm{~nm}$ light (Fig. 3b). The amplitude of the photoactivatable current was $16.1 \pm 2.1 \%$ (mean \pm s.e.m., $n=15$ ) of the saturating cholinergic current. The E61C mutation increased the $\mathrm{EC}_{50}$ of the $\alpha 4 \beta 2$ receptor for ACh. Labelling with MAACh did not change this $\mathrm{EC}_{50}$ further (Fig. 3d, Supplementary Table S1).

The prominence of the E61C mutant motivated us to carry out another molecular docking study, wherein the maleimide moiety of MAACh was 'virtually tethered' to be in the proximity of the 
a

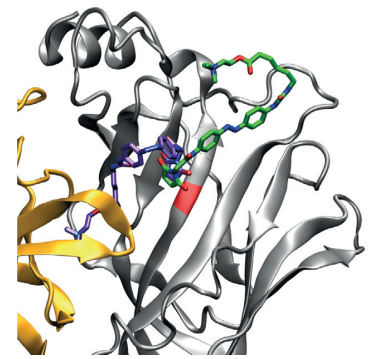

b

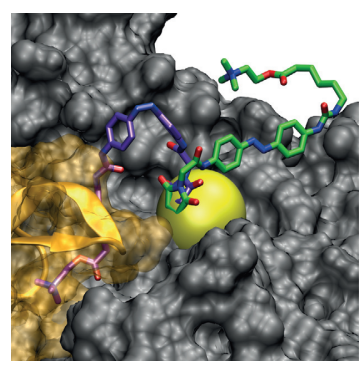

Figure 4 | Molecular model of a nAChR with a virtually tethered photoswitchable agonist. a,b, Docking results with constrained cis-MAACh (violet) and trans-MAACh (green) in the $\alpha 4 \beta 2$ homology model. The maleimide is constrained to be near the sulfhydryl group of C61. Ribbon (a) and surface (b) views of the minimum energy conformations are shown. The positional constraint (sphere) is bright yellow.

sulfhydryl group of the E61C residue (Fig. 4a,b). Allowing the remainder of the PTL to move freely resulted in docking of the ligand head group into the ligand-binding site in all conformations. By contrast, none of the tethered trans-MAACh conformations reached the ligand-binding site (Fig. 4a,b, Supplementary Fig. S5). These molecular docking studies corroborate our finding that the agonist is able to reach the ligand-binding site and activate the receptor only in its cis-form, and not in its trans-configuration.

Photoinhibition of engineered $\mathbf{n A C h R s}$. We then proceeded to test the effect of MAHoCh attached to the three cis-agonist active cysteine mutants in $\alpha 3 \beta 4$ heteromeric nAChRs. After treating oocytes with the PTL in the dark, we determined that illumination with $380 \mathrm{~nm}$ and $500 \mathrm{~nm}$ light did not produce photoactivatable currents with any of the three mutants (Supplementary Fig. S6). Instead, illumination with $380 \mathrm{~nm}$ light reduced dramatically the response of the $\alpha 3 \beta 4 \mathrm{E} 61 \mathrm{C}$ receptor to $\mathrm{ACh}$, and that with $500 \mathrm{~nm}$ light restored the initial response to ACh (Fig. 5a). This indicates that tethered MAHoCh acts as a cis-antagonist of the $\alpha 3 \beta 4 \mathrm{E} 61 \mathrm{C}$ receptor. ACh-evoked currents were not modulated substantially by illumination at the $\alpha 3 \beta 4 \mathrm{R} 113 \mathrm{C}$ and $\alpha 3 \beta 4 \mathrm{~S} 117 \mathrm{C}$ receptors (data not shown). To characterize the light-dependent block of the $\alpha 3 \beta 4 \mathrm{E} 61 \mathrm{C}$ receptor at different concentrations of $\mathrm{ACh}$, we compared the amount of current observed under $380 \mathrm{~nm}$ and $500 \mathrm{~nm}$ illumination for the same cells. The percentage of current blocked under $380 \mathrm{~nm}$ light ranged from $80 \pm 5 \%$ at $30 \mu \mathrm{M}$ ACh to $68 \pm 4 \%$ at a saturating $10 \mathrm{mM}$ ACh (mean \pm s.e.m., $n=5$ ) (Fig. 5c). Tethering MAHoCh at the $\alpha 4 \beta 2 \mathrm{E} 61 \mathrm{C}$ receptor produced a similar photoinhibition of the cholinergic current (Fig. 5b). The percentage of current blocked under $380 \mathrm{~nm}$ light at the $\alpha 4 \beta 2$ receptor ranged from $75 \pm 10 \%$ at $100 \mu \mathrm{M}$ ACh to $81 \pm 7 \%$ at a saturating $3 \mathrm{mM} \mathrm{ACh}$ (mean \pm s.e.m., $n=5$ ) (Fig. $5 \mathrm{~d}$ ).

In control experiments, we did not observe any photoactivation after treating oocytes that expressed either $\alpha 3 \beta 4 \mathrm{E} 61 \mathrm{C}$ or $\alpha 4 \beta 2 \mathrm{E} 61 \mathrm{C}$ nAChRs with AcAACh $(\mathbf{6 a}, \mathbf{b})$, a non-reactive analogue of MAACh (Supplementary Fig. S7). Furthermore, treatment of wild-type (WT) receptors with either MAACh or MAHoCh did not impart any photosensitivity (Supplementary Fig. S6). These results confirm that covalent modification at the genetically introduced cysteine residue is required for the optical control of the LinAChR function and suggest that off-target actions of these ligands at endogenous neuronal receptors should be minimal.

Thermal relaxation of the photoswitchable ligands. Azobenzene PTLs can be converted from their cis-configuration into their trans-configuration by $500 \mathrm{~nm}$ illumination, but they also spontaneously convert into the more stable trans-isomer through
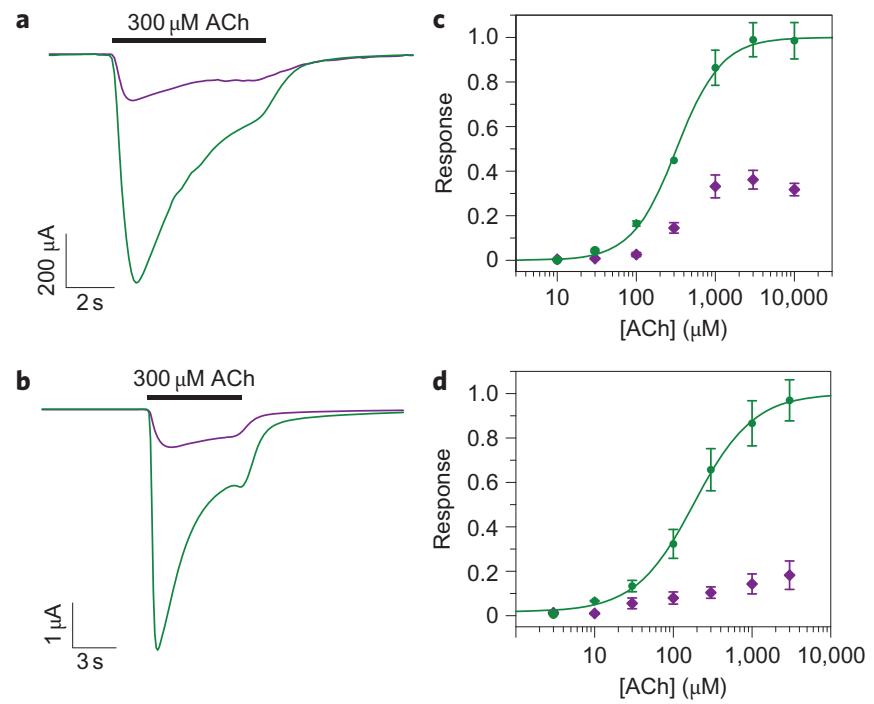

Figure 5 | Photoinhibition of nAChRs with a tethered antagonist. a,b, Photoinhibition of the cholinergic current of the $\alpha 3 \beta 4 \mathrm{E} 61 \mathrm{C}$ (a) and $\alpha 4 \beta 2 \mathrm{E} 61 \mathrm{C}$ (b) mutant receptors by tethered $\mathbf{M A H o C h}$. The current shown is in response to $300 \mu \mathrm{M}$ ACh under $380 \mathrm{~nm}$ light (violet lines) and $500 \mathrm{~nm}$ light (green lines). c,d, ACh dose-response curves for the $\alpha 3 \beta 4 \mathrm{E} 61 \mathrm{C}$ (c) mutant and the $\alpha 4 \beta 2 \mathrm{E} 61 \mathrm{C}$ (d) mutant with tethered MAHoCh under $500 \mathrm{~nm}$ light (green lines) and $380 \mathrm{~nm}$ light (violet lines). Data are mean \pm s.e.m., $n=5$.

thermal relaxation in the dark ${ }^{26}$. Thus, the MAACh-labelled $\alpha 4 \beta 2 \mathrm{E} 61 \mathrm{C}$ receptor can be activated by $380 \mathrm{~nm}$ light and the photoactivatable current decays over time in the dark (Fig. 6a). To investigate whether tethering the PTL to a receptor affects the rate of cis-isomer thermal relaxation, we compared the half-life of MAACh in solution, measured by changes in absorption at $380 \mathrm{~nm}$, to the calculated half-life of the decay of the photoactivatable current (Fig. 6b). The half-life of MAACh in solution was 28 seconds, close to the previously reported value of 26 seconds for a urea-azobenzene derivative ${ }^{27}$. In contrast, the thermal decay of the photoactivatable current could only be fitted to a biexponential decay curve, with a fast half-life of 4.7 seconds and a slow half-life of 94 seconds, which indicates that ligand binding increases the stability of cis-MAACh. The MAHoChlabelled $\alpha 4 \beta 2 \mathrm{E} 61 \mathrm{C}$ receptor can be blocked by $380 \mathrm{~nm}$ light and the amount of block also decays slowly in the dark (Fig. 6c). We measured the half-life of cis-MAHoCh in solution to be 74.4 minutes (Fig. 6d). The long half-life of cis-MAHoCh can be attributed to the alkoxy substituent on the azobenzene, which does not destabilize the cis-configuration to the same extent as the urea substituent in $\mathbf{M A A C h}{ }^{26}$. The thermal relaxation rate of tethered cis-MAHoCh appears to be of the same order of magnitude as that of cis-MAHoCh in solution.

\section{Discussion}

In a classic set of studies carried out in the late 1970s and early 1980s, Erlanger and co-workers demonstrated that azobenzene photoswitches could provide photosensitivity to endogenous muscle $\mathrm{nAChRs}{ }^{28,29}$. These pentameric ligand-gated ion channels naturally possess a disulfide bond at the tip of the so-called C-loops in their $\alpha$-subunits ${ }^{1}$, which is in close proximity to the ligand-binding site. This disulfide could be reduced to provide a reactive thiol for the covalent attachment of a PTL, although such treatment also reduced the affinity of the receptor for agonists by up to $100-$ fold $^{30}$. The PTL, called QBr, consisted of a benzylic bromide (the electrophile), an azobenzene photoswitch and a benzylic trimethylammonium moiety. Once attached, QBr activated 

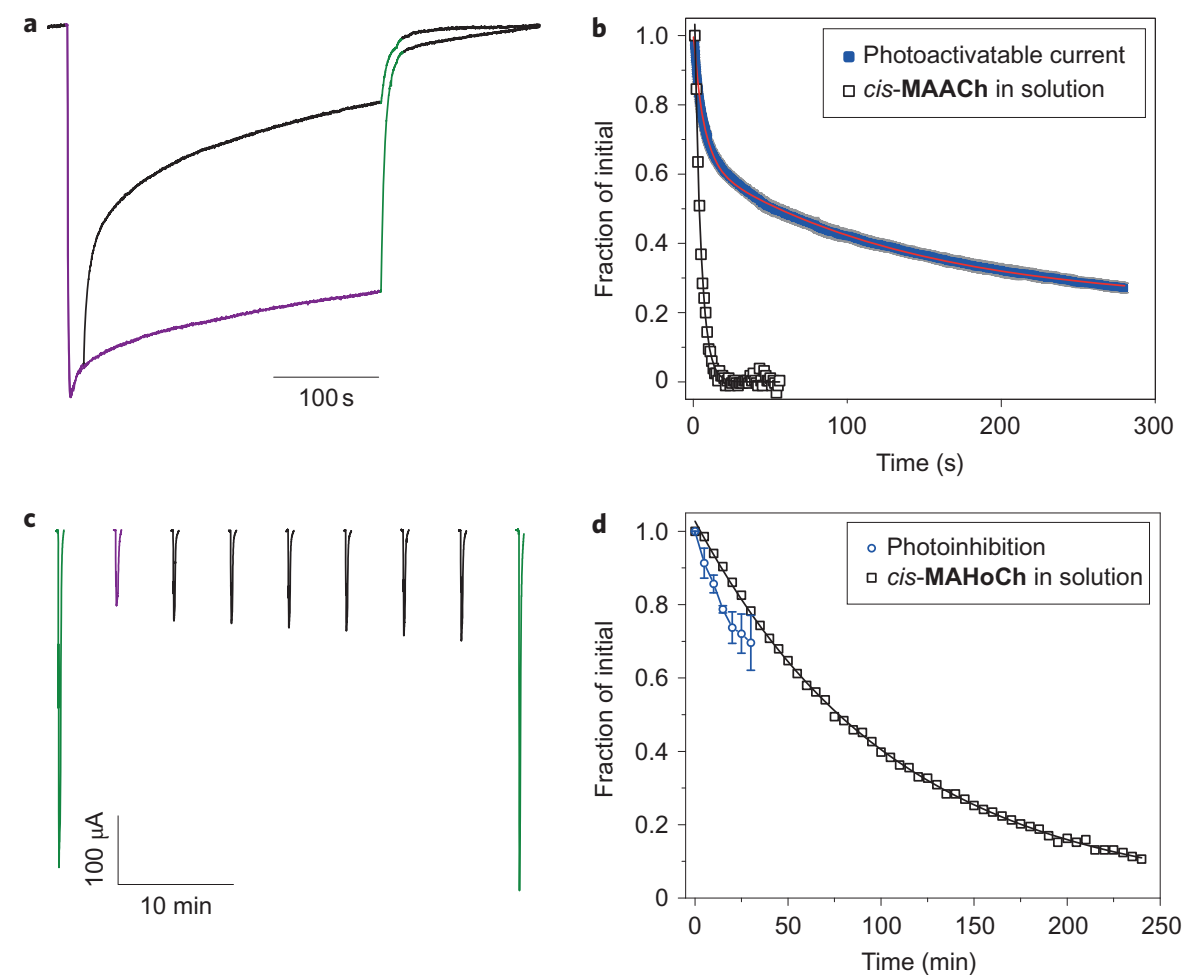

Figure 6 | Thermal relaxation of the photoswitchable agonist and antagonist. a, Normalized photoactivatable current decay of the $\alpha 4 \beta 2 \mathrm{E} 61 \mathrm{C}$ receptor in the dark with tethered MAACh. Decay of the photoactivatable current over time under $380 \mathrm{~nm}$ illumination (violet line) and in the dark (black line) is shown. b, Thermal relaxation of cis-MAACh in phosphate-buffered saline (open squares) fitted with a monoexponential decay function (black line) (half-life $\left(t_{1 / 2}\right)=28$ seconds). Decay of the photoactivatable current (mean (blue line) \pm s.e.m. (grey line), $n=4$ ) fitted using a biexponential decay function (red line, $t_{1 / 2}(1)=4.7$ seconds, $t_{1 / 2}(2)=94$ seconds). Both measurements are normalized. c, Decay of the photoinhibition of cholinergic current of the $\alpha 4 \beta 2 \mathrm{E} 61 \mathrm{C}$ receptor in the dark with tethered MAHoCh measured in Xenopus oocytes. ACh (100 $\mu \mathrm{M})$ was perfused under $500 \mathrm{~nm}$ light (green line), $380 \mathrm{~nm}$ (violet) light (violet line) and in the dark (black line). d, Thermal relaxation of cis-MAHoCh in phosphate-buffered saline (open squares) fitted with a monoexponential decay function (black line) ( $t_{1 / 2}=74.4$ minutes). Decay of the photoinhibition of cholinergic current of the $\alpha 4 \beta 2 \mathrm{E} 61 \mathrm{C}$ receptor with MAHoCh (blue line) is shown (mean \pm s.e.m., $n=4$ ).

the channel in its trans-form, that is at $500 \mathrm{~nm}$ or in the dark, but could be switched to an inactive cis-form by irradiation with $330 \mathrm{~nm}$ light. The resulting light-activated $\mathrm{nAChR}$ could be used to stimulate reversibly Electrophorus electroplaques, frog muscles and rat myoballs with light ${ }^{28,29,31,32}$. At the time these studies were carried out, however, receptors could not be manipulated genetically and expressed heterologously, so the impact of synthetic photoswitches on neuroscience research remained limited.

As a result of the enormous progress of molecular biology since the 1980s, it is now possible to express heterologously mutant receptors in Xenopus oocytes, mammalian cells, neurons and even transgenic animals $12,13,33$. Once expressed in a cell, our engineered LinAChR (E61C) can be conjugated with either an agonist or an antagonist, which provides a powerful bi-directional control of receptor activity. Both variants of LinAChR behave like normal $\mathrm{nAChRs}$ in the dark, but on irradiation with $380 \mathrm{~nm}$ they can be turned on in the absence of ACh or off in the presence of the neurotransmitter. The red-shifted PTL action spectra (compared to that of $\mathrm{QBr}$ ) should also minimize potential phototoxic effects. In principle, their spectral sensitivity could be moved further towards lower energies with appropriate substitution of the azobenzene photoswitch $^{34,35}$.

Another field that has undergone remarkable progress in recent years is the structural biology of pentameric ligand-gated ion channels (Cys-loop receptors) ${ }^{36}$. Several X-ray structures of homopentameric ligand-gated ion channels are now available ${ }^{37,38}$, as are highresolution structures of AChBPs bound to a variety of agonists and antagonists ${ }^{20,22}$. Without these, it would be difficult to design functional PTLs and choose appropriate sites for their attachment. The X-ray structure of carbamylcholine bound to AChBP and the homology models of the neuronal receptors ${ }^{20,21}$ proved to be most relevant for our design and computational evaluation of PTLs, and were particularly helpful for identifying residues suited to the attachment of the photoswitchable agonist MAACh. Our molecular docking studies correctly identified positions 61 and 117 as suitable sites of attachment. However, the predicted site 63 turned out to be unsuitable, which highlights the importance of functional screening as a complement to in silico models.

The antagonist action of tethered MAHoCh is more difficult to explain based on the available X-ray structures, but it is consistent with the known pharmacology of nAChRs (see Fig. 1). It is also consistent with the 'foot-in-the-door' mechanism of nAChR antagonism, wherein an antagonist binds, but prevents the complete conformational contraction of the ligand-binding site required for receptor activation ${ }^{39}$. Apparently, the elongation of HoChPE with a diazene unit bearing an aryl group is sufficient to prevent the C-loop of the receptor $\alpha$-subunit from closing in sufficiently to activate the receptor. This also makes sense given the structural similarity of the azobenzene MAHoCh with the stilbene MG-624, and timethylammonium derivatives thereof, which also function as antagonists ${ }^{19}$. Our data demonstrate that once an attachment site is chosen, it is possible to convert a tethered photoswitchable agonist into a tethered photoswitchable antagonist by altering the structure of the tether and the ligand head group. This matches previous observations by Sullivan and Cohen, who established that elongation of a covalently attached agonist by a single methylene 
group could turn it into an antagonist ${ }^{40}$. Conversely, it might be possible to use the same tethered ligand and two different sites of attachment to achieve either photoagonism or photoantagonism ${ }^{40}$.

The diversity of neuronal nAChRs and their structural similarities complicates selective targeting of specific $\mathrm{nAChR}$ subtypes pharmacologically. This study reports the reversible photoactivation and inhibition of common neuronal $\alpha 4 \beta 2$ and $\alpha 3 \beta 4$ nAChRs using light following the genetically targeted conjugation of PTLs. The findings should enable the rational design of other light-regulated pentameric ligand-gated ion channels, homopentameric $\alpha 7$ nAChRs and neuromuscular nAChRs. The lessons learned in this study should also facilitate the design of photosensitive $\mathrm{GABA}_{\mathrm{A}}$ $\left(\gamma\right.$-aminobutyric acid), GlyRs, $5 \mathrm{HT}_{3}$ or $\mathrm{GluCl}$ receptors. Based on the recent X-ray structure of $\mathrm{GluCl}$ and our expertise with photoswitchable tethered glutamate derivatives ${ }^{13}$, for instance, it should be possible to develop a hyperpolarizing light-gated chloride channel ${ }^{41}$. It is also conceivable that photoswitchable tethered agonists and antagonists could be extended to muscarinic ACh receptors, which have not yet been characterized in atomic detail, but for which extensive pharmacology exists ${ }^{42}$.

In future physiological investigations, LinAChRs will be expressed in dissociated neurons, intact neuronal tissues and live animals. We expect them to function well in these systems, perhaps even better than in Xenopus oocytes, the opacity of which makes it impossible to photoregulate receptors on the entire surface of the cell. In any case, the amount of photoactivation should be sufficient to replicate the major type of cholinergic transmission in the mammalian brain. Neuronal nAChRs are mostly extrasynaptic and respond to low micromolar concentrations of ACh diluted after synaptic release (that is, volume transmission ${ }^{43}$. Expression of the cysteine-containing subunit alone should produce functional LinAChRs in neurons that possess endogenous nicotinic receptors, which would then allow optical manipulation of the receptor function in vitro and in vivo ${ }^{44}$. Alternatively, native nAChR subunits can be replaced by cysteine mutants using a knock-in strategy ${ }^{45}$, which would preserve the endogenous pattern and level of receptor expression. We intend to pursue these strategies to study the physiological and pathological roles of heteromeric nAChRs in the brain and periphery and will report the results of these investigations in due course.

\section{Methods}

Synthesis of MAACh and MAHoCh. For this synthesis see the Supplementary Information and Supplementary Fig. S1.

Molecular cloning. Rat nAChR subunit complementary (deoxy)ribonucleic acids (cDNAs) in the pNKS2 vector (provided by A. Nicke, Max Planck Institute for Brain Research, Frankfurt am Main, Germany) were used for all experiments. To minimize non-specific labelling, the C-terminal $\alpha 4 \mathrm{C} 594$ and the $\beta 4 \mathrm{C} 75$ residues were both mutated to serine (mutations that do not affect the function of the receptor) ${ }^{46}$. These mutants were then used as a background for screening PTL-attachment sites. For nomenclature simplicity, the WT receptors are named in this article as $\alpha 3 \beta 4 \mathrm{WT}$ and $\alpha 4 \beta 2 \mathrm{WT}$, whereas the $\alpha 4 \mathrm{C} 594 \mathrm{~S} \beta 2$ and the $\alpha 3 \beta 4 \mathrm{C} 75 \mathrm{~S}$ background receptors are named $\alpha 4 \beta 2$ and $\alpha 3 \beta 4$, respectively. DNA was linearized with XbaI and ribonucleic acid (RNA) was transcribed using the mMessage mMachine SP6 transcription kit (Ambion). Surgically extracted X. laevis oocytes were injected with 2-20 ng of channel RNA (50 nl). The cells were incubated in oocyte Ringer's solution $(\mathrm{NaCl}$ (96 mM), $\mathrm{KCl}(2 \mathrm{mM}), \mathrm{CaCl}_{2}(1.8 \mathrm{mM}), \mathrm{MgCl}_{2}(1 \mathrm{mM})$ and HEPES (5 mM, $\mathrm{pH} 7.4)$ ) at $18{ }^{\circ} \mathrm{C}$ for $24-48$ hours before the experiments.

Molecular modelling. Molecular models of $\alpha 4 \beta 2$ neuronal nAChR (PDB ID:1ole) and of the T. californica nAChR (ref. 16) were used to identify endogenous cysteine residues accessible from the extracellular medium ${ }^{21}$. The X-ray structure of AChBP in complex with carbamylcholine ${ }^{20}$ was used to estimate the distance between the ammonium group of the ligand and the beta carbon of the engineered cysteine residues. The numbering of the residues in the homology model is not consistent with the protein sequence (see Supplementary Fig. S3 for the sequence alignment and residue numbering). Cis- and trans-ligands were docked to the homology model of the $\alpha 4 \beta 2$ receptor after the addition of hydrogen atoms and the removal of nonprotein moieties using Glide 5.7 as implemented in Maestro 9.2 (ref. 47). The docking consisted of a spatial fit of the ligand to the receptor grid, followed by minimization and scoring of hits based on a discretized ChemScore function ${ }^{48,49}$. Ligands were docked flexibly to a rigid receptor using standard precision and the top hits were examined. Docking of the constrained ligands contained an additional positional constraint that restricted the alkenyl carbons of the maleimide moiety to a shell with an inner radius of $1 \AA$ and an outer radius of $3.5 \AA$ centred at the sulfhydryl group of the C61 cysteine residue.

Photoswitch conjugation. All photoswitch compounds were dissolved in dimethylsulfoxide (DMSO) to make a stock solution $(10 \mathrm{mM})$ and diluted in oocyte Ringer's solution (see above) to $25 \mu \mathrm{M}$ for conjugation. For all recordings, the DMSO concentration was $<0.25 \%$ (volume/volume). Oocytes were incubated in the photoswitch compound solution for 20 minutes in the dark at room temperature before electrophysiological recordings. Photoswitch conjugation was followed by a five minute wash in oocyte Ringer's solution.

Electrophysiology. All electrophysiology experiments were conducted at room temperature. Two-electrode voltage-clamp experiments were performed using an OC-725C amplifier (Warner Instruments), DigiData 1200 interface and pClamp 8.0 software. The oocytes were placed in a perfusion chamber for the recordings. All the recordings were performed in oocyte Ringer's solution (see above). For each experiment, the oocyte membrane potential was held at $-80 \mathrm{mV}$. An eight-line perfusion system with a VC-8 valve controller (Warner Instruments) was used for perfusion of ACh into the chamber. Data were sampled at $1 \mathrm{kHz}$ and filtered at $10 \mathrm{~Hz}$. Cells were illuminated using a Lambda-LS illuminator that contained a $125 \mathrm{~W}$ xenon arc lamp (Sutter Instruments) equipped with narrow $( \pm 10 \mathrm{~nm})$ band-pass filters. The incident light intensity was $20 \mathrm{~mW} \mathrm{~cm}^{-2}$ for both $380 \mathrm{~nm}$ and $500 \mathrm{~nm}$ light, measured at the aperture using a handheld optical power meter (1918-C, Newport).

Data analysis. Dose-response curves were created using equation (1):

$$
y=A_{1}+\left(A_{2}-A_{1}\right) /\left(1+10^{\left(\log \left(x_{0}-x\right) \times p\right)}\right)
$$

where $p=$ the Hill coefficient, $10^{\log x_{0}}=\mathrm{EC}_{50}, A_{1}$ is the bottom asymptote and $A_{2}$ is the top asymptote of the dose-response curve.

Absorption spectra. Ultraviolet-visible spectra of the photoswitch compounds were obtained using the SmartSpec Plus Spectrophotometer (BioRad Laboratories) in PBS at $\mathrm{pH}$ 7.4. The change in absorbance of the compounds at $380 \mathrm{~nm}$ with time after photoconversion to the cis-configuration was used to determine the kinetics of thermal relaxation. The light source used for photoconversion of the ligands was the same as that used in electrophysiological recordings.

Received 17 August 2011; accepted 21 November 2011; published online 10 January 2012

\section{References}

1. Albuquerque, E. X., Pereira, E. F. R., Alkondon, M. \& Rogers, S. W. Mammalian nicotinic acetylcholine receptors: from structure to function. Physiol. Rev. 89, 73-120 (2009)

2. Kew, J. N. C. \& Davies, C. H. Ion Channels: From Structure To Function 2nd edn (Oxford Univ. Press, 2010).

3. Neher, E. \& Sakmann, B. Single-channel currents recorded from membrane of denervated frog muscle fibres. Nature 260, 799-802 (1976).

4. Unwin, N. Refined structure of the nicotinic acetylcholine receptor at $4 \AA$ resolution. J. Membr. Biol. 346, 967-989 (2005).

5. Nowak, M. W. et al. Nicotinic receptor binding site probed with unnatural amino acid incorporation in intact cells. Science 268, 439-442 (1995).

6. Noda, M. et al. Primary structure of alpha-subunit precursor of Torpedo californica acetylcholine receptor deduced from cDNA sequence. Nature 299, 793-797 (1982).

7. Noda, M. et al. Structural homology of Torpedo californica acetylcholinereceptor subunits. Nature 302, 528-532 (1983).

8. Noda, M. et al. Primary structures of beta-subunit and delta-subunit precursors of Torpedo californica acetylcholine-receptor deduced from cDNA sequences. Nature 301, 251-255 (1983).

9. Taly, A., Corringer, P-J., Guedin, D., Lestage, P. \& Changeux, J-P. Nicotinic receptors: allosteric transitions and therapeutic targets in the nervous system. Nat. Rev. Drug Discov. 8, 733-750 (2009).

10. Kramer, R. H., Fortin, D. L. \& Trauner, D. New photochemical tools for controlling neuronal activity. Curr. Opin. Neurobiol. 19, 544-552 (2009).

11. Fehrentz, T., Schönberger, M. \& Trauner, D. Optochemical genetics. Angew. Chem. Int. Ed. http://dx.doi.org/10.1002/anie.201103236 (in the press).

12. Banghart, M., Borges, K., Isacoff, E., Trauner, D. \& Kramer, R. H. Light-activated ion channels for remote control of neuronal firing. Nat. Neurosci. 7, 1381-1386 (2004)

13. Volgraf, M. et al. Allosteric control of an ionotropic glutamate receptor with an optical switch. Nat. Chem. Biol. 2, 47-52 (2006). 
14. Fortin, D. L. et al. Optogenetic photochemical control of designer $\mathrm{K}^{+}$channels in mammalian neurons. J. Neurophysiol. 106, 488-496 (2011).

15. Szobota, S. et al. Remote control of neuronal activity with a light-gated glutamate receptor. Neuron 54, 535-545 (2007).

16. Mourot, A. et al. Probing the reorganization of the nicotinic acetylcholine receptor during desensitization by time-resolved covalent labeling using [3H]AC5, a photoactivatable agonist. Mol. Pharmacol. 69, 452-461 (2006).

17. Hunt, R. \& Renshaw, R. R. Some effects of derivatives of betaine amide and of choline ethers on the autonomic nervous system. J. Pharmacol. Exp. Ther. 35, 99-128 (1929).

18. Wong, K. C. \& Long, J. P. Nicotinic and muscarinic activity of phenacyl and phenylalkyl trimethylamines. J. Pharmacol. Exp. Ther. 137, 70-75 (1962).

19. Gotti, C. et al. 4-Oxystilbene compounds are selective ligands for neuronal nicotinic Gk alpha-Bungarotoxin receptors. Br. J. Pharmacol. 124, 1197-1206 (1998).

20. Celie, P. H. N. et al. Nicotine and carbamylcholine binding to nicotinic acetylcholine receptors as studied in AChBP crystal structures. Neuron 41, 907-914 (2004).

21. Le Novère, N., Grutter, T. \& Changeux, J-P. Models of the extracellular domain of the nicotinic receptors and of agonist- and $\mathrm{Ca}^{2+}$-binding sites. Proc. Natl Acad. Sci. USA 99, 3210-3215 (2002).

22. Brejc, K. et al. Crystal structure of an ACh-binding protein reveals the ligand-binding domain of nicotinic receptors. Nature 411, 269-276 (2001).

23. Hansen, S. B. et al. Structures of Aplysia AChBP complexes with nicotinic agonists and antagonists reveal distinctive binding interfaces and conformations. EMBO J. 24, 3635-3646 (2005).

24. Chavez-Noriega, L. E. et al. Pharmacological characterization of recombinant human neuronal nicotinic acetylcholine receptors ha2b2, ha2b4, ha3b2, ha3b4, ha4b2, ha4b4 and ha7 expressed in Xenopus oocytes. J. Pharmacol. Exp. Ther. 280, 346-356 (1997).

25. Gotti, C., Zoli, M. \& Clementi, F. Brain nicotinic acetylcholine receptors: native subtypes and their relevance. Trends Pharmacol. Sci. 27, 482-491 (2006).

26. Nishimura N. et al. Thermal cis-to-trans isomerization of substituted azobenzenes II. Substituent and solvent effects. Bull. Chem. Soc. Jpn 49, 1381-1387(1976).

27. Pozhidaeva, N., Cormier, M. E., Chaudhari, A. \& Woolley, G. A. Reversible photocontrol of peptide helix content: adjusting thermal stability of the cis state. Bioconjug. Chem. 15, 1297-1303 (2004).

28. Bartels, E., Wassermann, N. H. \& Erlanger, B. F. Photochromic activators of the acetylcholine receptor. Proc. Natl Acad. Sci. USA 68, 1820-1823 (1971).

29. Lester, H. A., Krouse, M. E., Nass, M. M., Wassermann, N. H. \& Erlanger, B. F. A covalently bound photoisomerizable agonist: comparison with reversibly bound agonists at Electrophorus electroplaques. J. Gen. Physiol. 75, 207-232 (1980).

30. Barrantes, F. J. Modulation of acetylcholine receptor states by thiol modification. Biochemistry 19, 2957-2965 (1980).

31. Cox, R. N., Kawai, M., Karlin, A. \& Brandt, P. W. Voltage fluctuations at the frog sartorius motor endplate produced by a covalently attached activator. J. Membr. Biol. 51, 145-159 (1979).

32. Chabala, L. D. \& Lester, H. A. Activation of acetylcholine receptor channels by covalently bound agonists in cultured rat myoballs. J. Physiol. 379, 83-108 (1986).

33. Gorostiza, P. et al. Mechanisms of photoswitch conjugation and light activation of an ionotropic glutamate receptor. Proc. Natl Acad. Sci. USA 104, 10865-10870 (2007).

34. Sadovski, O., Beharry, A. A., Zhang, F. Z. \& Woolley, G. A. Spectral tuning of azobenzene photoswitches for biological applications. Angew. Chem. Int. Ed. 48, 1484-1486 (2009).
35. Mourot, A. et al. Tuning photochromic ion channel blockers. ACS Chem. Neurosci. 2, 536-543 (2011).

36. Corringer, P-J. et al. Atomic structure and dynamics of pentameric ligand-gated ion channels: new insight from bacterial homologues. J. Physiol. 588, 565-572 (2010).

37. Bocquet, N. et al. A prokaryotic proton-gated ion channel from the nicotinic acetylcholine receptor family. Nature 445, 116-119 (2007).

38. Hilf, R. J. C. \& Dutzler, R. X-ray structure of a prokaryotic pentameric ligandgated ion channel. Nature 452, 375-379 (2008).

39. Law, R. J., Henchman, R. H. \& McCammon, J. A. A gating mechanism proposed from a simulation of a human alpha7 nicotinic acetylcholine receptor. Proc. Natl Acad. Sci. USA 102, 6813-6818 (2005).

40. Sullivan, D. A. \& Cohen, J. B. Mapping the agonist binding site of the nicotinic acetylcholine receptor. Orientation requirements for activation by covalent agonist. J. Biol. Chem. 275, 12651-12660 (2000).

41. Hibbs, R. E. \& Gouaux, E. Principles of activation and permeation in an anion-selective Cys-loop receptor. Nature 474, 54-60 (2011).

42. Langmead, C. J., Watson, J. \& Reavill, C. Muscarinic acetylcholine receptors as CNS drug targets. Pharmacol. Ther. 117, 232-243 (2008).

43. Dani, J. A. \& Bertrand, D. Nicotinic acetylcholine receptors and nicotinic cholinergic mechanisms of the central nervous system. Annu. Rev. Pharmacol. Toxicol. 47, 699-729 (2007).

44. Aravanis, A. M. et al. An optical neural interface: in vivo control of rodent motor cortex with integrated fiberoptic and optogenetic technology. J. Neural Eng. 4, S143-S156 (2007)

45. Thomas, K. R. \& Capecchi, M. R. Site-directed mutagenesis by gene targeting in mouse embryo-derived stem cells. Cell 51, 503-512 (1987).

46. Mourot, A., Bamberg, E. \& Rettinger, J. Agonist- and competitive antagonistinduced movement of loop 5 on the alpha subunit of the neuronal alpha4beta4 nicotinic acetylcholine receptor. J. Neurochem. 105, 413-424 (2008).

47. Friesner, R. A. et al. Glide: a new approach for rapid, accurate docking and scoring. 1. Method and assessment of docking accuracy. J. Med. Chem. 47, 1739-1749 (2004)

48. Eldridge, M. D., Murray, C. W., Auton, T. R., Paolini, G. V. \& Mee, R. P. Empirical scoring functions: I. The development of a fast empirical scoring function to estimate the binding affinity of ligands in receptor complexes. J. Comput. Aided Mol. Des. 11, 425-445 (1997).

49. Baxter, C. A., Murray, C. W., Clark, D. E., Westhead, D. R. \& Eldridge, M. D. Flexible docking using Tabu search and an empirical estimate of binding affinity. Proteins: Struct., Funct., Bioinf. 33, 367-382 (1998).

\section{Acknowledgements}

Support for the work was provided by the Nanomedicine Development Center for the Optical Control of Biological Function PN2EY018241 (D.T., R.H.K.), The European Research Commission Advanced Grant (D.T.) and the Deutsche Forschungsgemeinschaft SFB 749 (D.T.)

\section{Author contributions}

M.R.B., A.M., R.H.K. and D.T. designed the research. M.R.B. and J.Z.Y. synthesized the ligands. I.T., A.M. and B.G. performed the research and analysed the data. I.T., M.R.B. and D.T. co-wrote the paper.

\section{Additional information}

The authors declare no competing financial interests. Supplementary information and chemical compound information accompany this paper at www.nature.com/ naturechemistry. Reprints and permission information is available online at http://www. nature.com/reprints. Correspondence and requests for materials should be addressed to R.H.K. and D.T. 